\title{
La representación de la mujer en publicidad: (des)igualdad cuantitativa y cualitativa en la creatividad española ${ }^{1}$
}

\author{
Alejandra HERnÁNDEz RuIz \\ Universidad de Alicante \\ alejandra.hernandez@ua.es \\ Marta MARTín LLAGUNO \\ Universidad de Alicante \\ marta.martin@ua.es \\ Marina BELÉNDEZ VÁZQUEZ \\ Universidad de Alicante \\ marina.belendez@ua.es
}

Recibido: $27 / 07 / 2012$

Aceptado: 30/10/2012

\section{Resumen}

En nuestro país, las evidencias empíricas previas confirman la falta de una representación igualitaria y no discriminatoria de la mujer en el discurso publicitario. Por otra parte, los estudios sobre la situación de la mujer en la empresa publicitaria evidencian la infrarrepresentación de las féminas en los departamentos creativos. En este trabajo pretendemos analizar, desde una perspectiva novedosa, si existe alguna relación entre la pervivencia de estereotipos sexistas en la publicidad y las estructuras productivas de la industria publicitaria (con especial atención a la composición sexual de los departamentos creativos). A través de la técnica cuantitativa del análisis de contenido, adoptaremos como unidades de análisis los spots recopilados en los anuarios creativos de la revista Anuncios en el período (2008-2011).

Palabras clave: mujer, publicidad, género, creatividad, igualdad.

\section{The Representation of Women in Advertising: Quantitative and Qualitative Inequality in Spanish Creativity}

\begin{abstract}
In Spain, prior empirical evidence confirms the lack of equal and non-discriminatory representation of women in advertising discourse. Moreover, studies on the situation of women in advertising firms prove the underrepresentation of females in creative departments. In this paper, we have applied a novel method to analyse the possible existence of a relationship between the persistence of gender stereotypes in advertising and the production structures of advertising companies (with particular emphasis on the gender composition of creative departments). Using a quantitative content analysis technique, we studied spots included in the Anuncios creative magazine yearbooks during the period (2008-2011) as units of analysis.
\end{abstract}

Keywords: women, advertising, gender, creativity, equality.

\section{Referencia normalizada}

HERNÁNDEZ RUIZ, Alejandra; MARTÍN LLAGUNO, Marta y BELÉNDEZ VÁZQUEZ, Marina (2012): "La representación de la mujer en publicidad: (des)igualdad cuantitativa y cualitativa en la creatividad española". Estudios sobre el mensaje periodístico. Vol. 18, núm. especial noviembre, págs.: 521530. Madrid, Servicio de Publicaciones de la Universidad Complutense.

1 Este estudio forma parte del proyecto de investigación "Estructuras productivas y manifiestos publicitarios sexistas: ¿una relación simbiótica?”, financiado por el Ministerio de Ciencia e Innovación (FEM2009-13149). 
Sumario: 1. Los estudios sobre la presencia de la mujer en el discurso publicitario: roles, sexismo y sesgos de género. 2. Metodología. 3. Desarrollo. 4. Conclusiones. 5. Referencias bibliográficas.

\section{Los estudios sobre la presencia de la mujer en el discurso publicitario: roles, sexismo y sesgos de género}

En la producción científica sobre la presencia de estereotipos de género en los medios de comunicación, y en particular, en el medio televisivo, uno de los protocolos de codificación más utilizados es el de McArthur y Resko (1975), centrado en cuantificar, entre otras cuestiones, el número de mujeres y hombres protagonistas, los roles desempeñados, la localización, la credibilidad de los personajes, las recompensas obtenidas, etc. A partir de este trabajo pionero, estudios ulteriores han replicado esta metodología en diversos países. En la revisión de Furnham y Mak (1999) se evidencia que en los 14 estudios que en 11 países han aplicado los indicadores cuantitativos de McArthur y Resko (1975) los hombres aparecen con más frecuencia que las mujeres en el rol de profesional o entrevistador, mientras que las mujeres destacan en los roles dependientes (madre, esposa, ama de casa). En este sentido, las mujeres aparecen confinadas al hogar con más frecuencia que los hombres y éstos predominan en las localizaciones propias del ocio y en la categoría "otros". Sin embargo, no hay diferencias significativas en el "lugar de trabajo" como localización.

En nuestro país, las evidencias empíricas previas confirman que los estereotipos más tradicionales de mujer y de hombre siguen vigentes. Así, Berganza y Del Hoyo (2006) al aplicar el protocolo de codificación de McArthur y Resko (1975) muestran cómo un $40 \%$ de los personajes femeninos analizados continúa asumiendo funciones dependientes (en los roles de ama de casa, esposa, e incluso objeto sexual), frente al $9,4 \%$ de los personajes masculinos que desempeñan estos roles. Los hombres destacan como entrevistadores/narradores o profesionales (en el 72,3\% de los casos), mientras que las mujeres sólo detentan el $35 \%$ de estos roles.

Por otro lado, por el objeto de nuestro estudio, destaca la propuesta de Pingree, Hawkins, Butler y Paisley (1976). A diferencia de la investigación desarrollada hasta el momento, para estos autores, es necesario abordar el sexismo en los medios a partir de indicadores cualitativos que claramente determinen si en publicidad la representación del género sugiere que mujeres y hombres no tienen el mismo valor o no deberían disfrutar de los mismos derechos (Utterström, 1977). El instrumento de evaluación de Pingree et al. (1976) está formado por una escala de cinco niveles que, siguiendo el trabajo de Royo-Vela, Aldás-Manzano, Küster y Vila (2008), representan un sexismo alto (1 y 2), moderado (3) o bajo (4 y 5).

Los trabajos de Royo-Vela, Aldás-Manzano, Küster-Boluda y Vila-Lopez (2007) y Royo-Vela et al. (2008) constituyen las únicas referencias empíricas que, en nuestro país y, hasta la fecha, han incorporado la perspectiva de Pingree et al. (1976) al análisis de la representación de hombres y mujeres en publicidad. Los resultados difieren en función del medio analizado. Así, en la investigación longitudinal de Royo-Vela et al. (2007) sobre los estereotipos de género en la publicidad gráfica durante un lapso de 24 años (1975-1999), las representaciones en los valores extremos (1 y 5) han desaparecido, de modo que la mayoría de las imágenes de hombres $(78,5 \%)$ y mujeres 
(67\%) pertenecen al nivel 4 (bajo sexismo). Sin embargo, aún existe un 21,5\% de mujeres y un $31,1 \%$ de hombres representados en el nivel 2 (alto sexismo).

Por su parte, la investigación de Royo-Vela et al. (2008), centrada en el medio televisivo, muestra que las mujeres son representadas con más frecuencia que los hombres en las posiciones extremas de la escala (nivel $1: 25 \%$ de mujeres vs. $4 \%$ de hombres; nivel $5: 17 \%$ de mujeres vs. $3 \%$ de hombres). Sin embargo, los hombres (98\%) aparecen representados con más frecuencia que las mujeres en el nivel $2(82 \%)$, en la categoría de alto sexismo. Por tanto, en televisión, y a partir de los resultados descritos, hombres y mujeres son representados de forma más sexista que en la publicidad gráfica.

Por otro lado, en nuestro país, por la novedad de su planteamiento y su utilidad para detectar inequidades es preciso mencionar la propuesta del sesgo de género, un instrumento de evaluación que compendia indicadores cuantitativos y cualitativos para analizar la (des)igualdad.

En concreto, este protocolo de evaluación se gesta en 2004 en el Departamento de enfermería comunitaria, medicina preventiva y salud pública e historia de la ciencia de la Universidad de Alicante, auspiciada por miembros del grupo de investigación de Salud Pública (Ruiz-Cantero, Martín-Llaguno, La Parra, Vives y Albaladejo, 2004). Así, para estos autores, y en el caso particular de las noticias de salud, en general, el sesgo se produce "siempre y cuando uno de los dos sexos reciba un tratamiento informativo perjudicial, en especial, cuando se compara con el recibido por el otro sexo" (Ruiz-Cantero et al., 2004: 67). Una vez establecido el concepto de sesgo de género, se establecieron tres indicadores para operacionalizar este constructo: visibilidad, paridad y empoderamiento.

Del trabajo de Ruiz-Cantero et al. (2004) se desprende que el término "visibilidad" trata de medir la igualdad entre sexos, en términos cuantitativos. Así, el interés reside en cuantificar el número de hombres y de mujeres (en términos absolutos o relativos) presentes en el mensaje informativo. Muy relacionado con este concepto, con la "paridad" se focaliza la atención en la igualdad cualitativa entre mujeres y hombres, de modo que ambos sexos deben ser representados de forma equilibrada en acciones como decir, hacer y en su presentación como sujetos pasivos. Por último, con el constructo "empoderamiento" el objetivo consiste en detectar si existe un equilibrio entre sexos en su representación como decisores o acatadores de órdenes.

En el ámbito concreto de la publicidad, con el fin de analizar la representación cuantitativa y cualitativa de hombres y mujeres en la publicidad de fármacos, la propuesta del sesgo de género se utilizó de forma pionera en el trabajo de Cambronero, Ruiz y Papí (2009). Los hallazgos más relevantes de esta investigación confirman que no existe ni paridad ni empoderamiento en la representación de hombres y mujeres en los anuncios de fármacos psicotrópicos y cardiovasculares. Así, no existe equidad en el desempeño de tareas o funciones y las mujeres son mostradas en posiciones de subordinación con mayor frecuencia que los hombres. Por otra parte, las mujeres están sobrerrepresentadas en los psicotrópicos y los hombres hacen lo propio en los cardiovasculares.

Finalmente, desde una perspectiva intercultural, Martín-Llaguno y Navarro (2012) retoman esta operacionalización para detectar el sexismo de una muestra de anuncios españoles y argentinos presentados en el Festival Iberoamericano de la Publicidad en su edición de 2009. De forma sucinta, en esta investigación se detecta que a pesar de 
los esfuerzos legislativos para erradicar la violencia simbólica en la publicidad, el sexismo publicitario sigue estando presente en ambos países. Así, la visibilidad de los varones es superior a la de las féminas. Del mismo modo, tampoco existe paridad entre sexos, ya que los hombres dicen y padecen acciones con más frecuencia que las mujeres que, por su parte, hacen en mayor número que los hombres. En cuanto al empoderamiento, en España existe el mismo porcentaje de hombres y de mujeres que toman y acatan decisiones, mientras que en Argentina las mujeres deciden con más frecuencia que los hombres que, en mayor medida, asumen las decisiones adoptadas.

Por otra parte, junto a la falta de una representación igualitaria y no discriminatoria de la mujer como objeto del discurso publicitario, los estudios sobre la situación de la mujer en la empresa publicitaria en nuestro país constatan que la presencia de las féminas en los departamentos creativos (responsables del desarrollo, creación y producción de ideas) es ínfima (Martín-Llaguno et al., 2007; Pueyo, 2010; García-González y Piñeiro-Otero, 2011).

A pesar de la profusión de estudios que analizan la publicidad con enfoque de género, en este trabajo nos planteamos, desde una perspectiva novedosa, como objetivo general, observar si existe alguna relación entre la composición sexual de los departamentos creativos (responsables de la ideación y concepción de las campañas publicitarias) y el nivel de sexismo de los mensajes publicitarios. Para la consecución de este objetivo general nos proponemos los siguientes objetivos específicos:

a) Cuantificar el número de hombres y de mujeres que componen los departamentos creativos;

b) Observar el rol desempeñado por las figuras masculinas y femeninas en los spots;

c) Analizar el nivel de sexismo de los anuncios;

d) Observar la (des)igualdad cuantitativa de hombres y mujeres representados de forma audiovisual, visual y sonora;

e) Detectar si existe paridad en la representación de ambos sexos;

f) Examinar si existen diferencias en el empoderamiento de hombres y mujeres en publicidad.

\section{Metodología}

En nuestro trabajo utilizamos la técnica cuantitativa del análisis de contenido y adoptamos, como unidades de análisis, los spots recopilados en los anuarios creativos de la revista Anuncios en el período (2008-2011). Las revistas especializadas constituyen una fuente de consulta especialmente útil para los objetivos de nuestra investigación, ya que proporcionan información sobre los profesionales de la publicidad implicados en las piezas publicitarias seleccionadas para su publicación. En particular, la revista Anuncios, de periodicidad semanal, con una circulación de 4000 ejemplares, publica cada año un anuario que compendia los spots representativos de la mejor creatividad española del año precedente ${ }^{2}$. Esta recopilación refleja el mejor nivel de la creatividad

2 Las ediciones 2009, 2010, 2011 y 2012 del anuario pueden consultarse online en: http://www.anuncios.com/anuarios 
audiovisual y gráfica de nuestro país y responde a la publicidad real, alejada de festivales y competiciones creativas. Para nuestra investigación seleccionamos los anuncios de televisión y descartamos las piezas de cine, websites y gráficas. Del total de 1322 piezas recopiladas, tras eliminar los spots sin posibilidad de visualización (a causa de la inoperatividad del enlace) y los anuncios cuya ficha técnica no correspondía a las características del spot en cuestión, obtuvimos un corpus objeto de análisis de 1315 spots.

Para la codificación de las figuras masculinas y femeninas se determinó seleccionar los dos primeros personajes centrales que constituyeran el foco de interés visual y verbal. De los 1315 anuncios objeto de estudio, en 1124 piezas (el 85,5\% de la muestra total) fue posible codificar, al menos, un personaje. El $26,1 \%$ corresponde a los anuncios de 2008 (edición de 2009), el 27,6\% pertenece a 2009 (edición de 2010), el $25,1 \%$ concierne a 2010 (edición de 2011) y el 21,3\% atañe a 2011 (edición de 2012).

El protocolo de codificación incluye, por una parte, variables relacionadas con las características generales de los anuncios como la ficha técnica de la pieza publicitaria, en la que se recogen datos como la agencia, el anunciante, el sector, el producto, la marca y el equipo profesional. En particular, para nuestro estudio, es de interés la composición sexual del departamento creativo. Para cuantificar el número de hombres y mujeres que forman parte del área de creación, se registraron todos los profesionales que, en la ficha técnica del spot analizado, ocuparan los siguientes puestos de trabajo: director/a general creativo/a, director/a creativo/a ejecutivo/a, director/a creativo/a, supervisor/a creativo/a, equipo creativo, director/a general de arte, director/a de arte, diseño, redactor/a, vicepresidente/a creativo/a, equipo arte, director/a general creativo/a digital, creativo/a y responsable creativo/a.

Por otra parte, la plantilla de codificación contempla las características demográficas, actitudinales y comportamentales de los personajes principales. Para analizar el rol desempeñado por las figuras femeninas y masculinas se utilizó la medida desarrollada por McArthur y Resko (1975) y se incorporaron algunas de las adaptaciones realizadas en estudios previos sobre el tema (Furnham y Bitar, 1993; Gilly, 1988; Kim y Lowry, 2005; Manstead y McCulloch, 1981; Moon y Chan, 2002; Neto y Pinto, 1988, Uray y Burnaz; 2003).

Por otro lado, para analizar el nivel de sexismo de las piezas publicitarias se seleccionó The Consciousness Scale for Sexism, un instrumento de evaluación desarrollado por Butler y Paisley (1974), aplicado por Pingree et al. (1976) al análisis de las imágenes sexistas de la mujer en publicidad y utilizado por otros autores en nuestro país (Royo-Vela et al., 2007 y Royo-Vela et al., 2008).

La escala está compuesta de cinco categorías que representan un sexismo alto (1 y 2), moderado (3) y bajo (4 y 5). Así, en la categoría 1 hombres y mujeres son tratados como objetos sexuales o decorativos; en el nivel 2 mujeres y hombres son mostrados en roles tradicionales (hombre en el trabajo, mujer en casa); en la categoría 3 hombres y mujeres desempeñan roles tradicionales y no tradicionales, con predominio de los no tradicionales; en el nivel 4 mujeres y hombres son tratados como iguales, de forma que pueden desarrollar roles tradicionales y no tradicionales sin predominio de ninguno de ellos; en la categoría 5 no hay sexismo, de modo que no es 
posible hablar de roles tradicionales o no tradicionales.

Para examinar la "Visibilidad" se observó la igualdad cuantitativa de hombres y mujeres representados de forma audiovisual, visual y sonora.

Para el constructo "Paridad" se registró si hombres y mujeres en publicidad dicen, hacen y padecen.

Por último, para analizar el "Empoderamiento" se analizó si mujeres y hombres son representados como decisores o acatadores de órdenes.

La codificación fue realizada por un equipo mixto formado por egresadas y egresados en Publicidad y Relaciones Públicas (dos mujeres y dos hombres) que previamente fueron entrenados en el uso del código y en la metodología del análisis de contenido. El coeficiente de acuerdo se realizó con una submuestra piloto del $15 \%$ de la muestra total, fue calculado dividiendo el número de acuerdos de los codificadores entre el total de ítems (anuncios) examinados y tuvo un valor de un $0,93 \%$. Las discrepancias entre los codificadores fueron resueltas a partir de la discusión y el diálogo.

\section{Desarrollo}

De las 1124 fichas técnicas codificadas, correspondientes a los spots con protagonistas, registramos un total de 1663 profesionales que pertenecen al departamento creativo, de los cuales, 1098 (66\%) son hombres y 565 (34\%) son mujeres.

Por lo que se refiere al rol desempeñado por las figuras masculinas y femeninas en los spots, en la tabla 1 se observa cómo la mayor parte de los casos analizados pertenecen a la categoría "otros", tanto para ellos $(34,5 \%)$ como para ellas (37\%). Aunque para ambos sexos el rol predominante es el de "profesional", los hombres superan a las mujeres en 15,58 puntos porcentuales. En sentido opuesto, las mujeres destacan sobre los hombres en el rol de "ama de casa" con una diferencia de 4,6 puntos porcentuales y, por último, la mujer aparece representada como "esposa", con más frecuencia que el hombre como "marido", con una diferencia de 4,17 puntos porcentuales.

Respecto a la representación de los personajes en roles dependientes (marido/mujer, padre/madre, hijo/a, novio/a, ama/o de casa, objeto sexual) e inde-

Tabla 1. Rol de las figuras masculinas y femeninas

\begin{tabular}{|c|c|c|c|c|c|c|c|}
\hline Sexo & Rol & Frecuencia & $\%$ & Sexo & Rol & Frecuencia & $\%$ \\
\hline Hombre & Marido & 83 & 6,84 & Mujer & Mujer & 84 & 11,01 \\
\hline & Padre & 60 & 4,94 & & Madre & 64 & 8,39 \\
\hline & Hijo & 90 & 7,42 & & Hija & 43 & 5,64 \\
\hline & Novio & 61 & 5,03 & & Novia & 56 & 7,34 \\
\hline & Amo de casa & 11 & 0,91 & & Ama de casa & 42 & 5,51 \\
\hline & Objeto sexual & 12 & 0,99 & & Objeto sexual & 19 & 2,49 \\
\hline & Profesional & 410 & 33,8 & & Profesional & 139 & 18,22 \\
\hline & $\begin{array}{c}\text { Entrevistador } \\
\text { /narrador }\end{array}$ & 68 & 5,61 & & $\begin{array}{l}\text { Entrevistador } \\
\text { a/narradora }\end{array}$ & 34 & 4,46 \\
\hline & Otros & 418 & 34,46 & & Otros & 282 & 36,96 \\
\hline & Total & 1213 & $100 \%$ & & Total & 763 & $100 \%$ \\
\hline
\end{tabular}

Fuente: elaboración propia 
pendientes (profesional, entrevistador/narrador), se observa un acercamiento entre ambos sexos. Así, las mujeres desempeñan roles dependientes en un $40,38 \%$ de los casos (frente al $26,13 \%$ de los hombres que hace lo propio). Por su parte, los hombres aparecen en la escena publicitaria ejerciendo roles independientes en el 39,41\% de las ocasiones, en contraposición al 22,68\% de mujeres que asume tales roles.

En cuanto al nivel de sexismo de los anuncios de nuestra muestra, la mayoría de los casos registrados pertenecen a la categoría de bajo sexismo, los niveles 4 y 5 . Así, en el $24,2 \%$ de la muestra objeto de estudio, hombres y mujeres son tratados como iguales, de forma que pueden desarrollar roles tradicionales y no tradicionales sin predominio de ninguno de ellos. Por su parte, en el 45,6\% de los casos no es posible hablar de roles tradicionales y no tradicionales, ya que hombres y mujeres no son juzgados en función de su género.

Por otra parte, el análisis de correlación bivariada no muestra ninguna relación significativa entre la composición sexual de los departamentos creativos y el nivel de sexismo (ver tabla 2).

Tabla 2. Correlación bivariada: nivel de sexismo y dptos. masculinos y femeninos

\begin{tabular}{|c|c|c|c|}
\hline & 1 & 2 & 3 \\
\hline $\begin{array}{l}\text { Nivel de sexismo } \\
\text { (variable inversa) }\end{array}$ & - & & \\
\hline $\begin{array}{l}\text { 2. Departamentos } \\
\text { creativos } \\
\text { masculinos }\end{array}$ & 021 & - & \\
\hline $\begin{array}{l}\text { 3. Departamentos } \\
\text { creativos } \\
\text { femeninos }\end{array}$ &,- 021 &,- 034 & \\
\hline
\end{tabular}

Fuente: elaboración propia

Con relación a los sesgos de género, la tabla 3 recoge las frecuencias y porcentajes de los casos en que mujeres y hombres aparecen en el mensaje publicitario de forma audiovisual, visual y sonora. Las pruebas de $\mathrm{X}^{2}$ ofrecen diferencias significativas entre los hombres y las mujeres que son representados de forma audiovisual $\left(\mathrm{X}^{2}=\right.$ $87,04 ; \mathrm{p}=0,00)$ y visual $\left(\mathrm{X}^{2}=29,33 ; \mathrm{p}=0,00\right)$, a favor de los primeros.

Tabla 3. Visibilidad

\begin{tabular}{|c|c|c|}
\hline Variable & Frecuencia & Porcentaje \\
\hline Hombres (audiovisual) & 549 & $48,8 \%$ \\
\hline Mujeres (audiovisual) & 388 & $34,5 \%$ \\
\hline Hombres (visual) & 889 & $79,1 \%$ \\
\hline Mujeres (visual) & 786 & $69,9 \%$ \\
\hline Hombres (audio) & 641 & $57,0 \%$ \\
\hline Mujeres (audio) & 346 & $30,8 \%$ \\
\hline
\end{tabular}

Fuente: elaboración propia

Por otro lado, en la tabla 4 se muestra el porcentaje de casos en que hombres y mujeres hacen, dicen y padecen. En este sentido, las pruebas de $\mathrm{X}^{2}$ evidencian que existen diferencias estadísticamente significativas entre los hombres y las mujeres que 
hacen $\left(X^{2}=57,36 ; p=0,00\right)$ y dicen $\left(X^{2}=19,03 ; p=0,00\right)$, a favor de los varones en ambos casos.

Tabla 4. Paridad

\begin{tabular}{|c|c|c|}
\hline Variable & Frecuencia & Porcentaje \\
\hline Hombres hacen & 756 & $67,3 \%$ \\
\hline Mujeres hacen & 591 & $52,6 \%$ \\
\hline Hombres dicen & 571 & $50,8 \%$ \\
\hline Mujeres dicen & 360 & $32,0 \%$ \\
\hline Hombres padecen & 187 & $16,6 \%$ \\
\hline Mujeres padecen & 114 & $10,1 \%$ \\
\hline
\end{tabular}

Fuente: elaboración propia

Finalmente, respecto al constructo "Empoderamiento", los análisis estadísticos confirman que existen diferencias significativas entre los hombres y las mujeres que toman $\left(X^{2}=56,33 ; p=0,00\right)$ y acatan decisiones $\left(X^{2}=144,56 ; p=0,00\right)$. Así, como se puede observar en la tabla 5 , los hombres destacan sobre las mujeres como decisores y acatadores de órdenes.

Tabla 5. Empoderamiento

\begin{tabular}{|c|c|c|}
\hline Variable & Frecuencia & Porcentaje \\
\hline Hombres toman decisiones & 300 & $26,7 \%$ \\
\hline Mujeres toman decisiones & 203 & $18,1 \%$ \\
\hline Hombres acatan decisiones & 107 & $9,5 \%$ \\
\hline Mujeres acatan decisiones & 59 & $5,2 \%$ \\
\hline
\end{tabular}

Fuente: elaboración propia

\section{Conclusiones}

En primer lugar, los resultados expuestos muestran que en la línea de los estudios realizados hasta el momento en nuestro país (Martín-Llaguno et al., 2007; Pueyo, 2010; García-González y Piñeiro-Otero, 2011), la mujer está infrarrepresentada en los departamentos creativos.

Sin embargo, a diferencia de las evidencias empíricas de Furnham y Mak (1999), en nuestro estudio, el rol predominante para ambos sexos (tras la categoría "otros") es el de profesional (aunque con más fuerza en el caso de los hombres). En el mismo sentido, y en contraposición a los datos de Berganza y Del Hoyo (2006), las diferencias entre sexos también se diluyen al considerar los roles dependientes (marido/mujer, padre/madre, hijo/a, novio/a, ama/o de casa, objeto sexual) e independientes (profesional, entrevistador/narrador).

En tercer lugar, con relación al nivel de sexismo de las piezas publicitarias analizadas, los resultados de nuestro estudio concuerdan parcialmente con los de RoyoVela et al. (2007). Así, las representaciones en el extremo inferior de la escala (la posición con un mayor sexismo) son ínfimas. En el mismo sentido, son escasos los anuncios pertenecientes al nivel 2. Es, por su parte, el nivel 5 (bajo sexismo) el que concentra el mayor porcentaje de casos. 
En cuarto lugar, la propuesta de sesgo de género revela que existe una desigualdad cuantitativa y cualitativa entre ambos sexos, a favor de los varones.

Finalmente, en el ámbito de los estudios que analizan la publicidad con enfoque de género, en esta investigación pretendíamos testar, desde una perspectiva novedosa, si existe alguna relación entre la composición sexual de los departamentos creativos y la presencia de sesgos sexistas en el mensaje publicitario. En este sentido, no existe ninguna relación significativa entre el sexo predominante en los departamentos creativos y el sexismo publicitario. Estudios ulteriores deberían analizar las creencias de género de los profesionales adscritos al área de creación y la identidad de marca de los productos objeto de publicidad para corroborar su posible repercusión en la generación de manifiestos publicitarios sexistas.

\section{Referencias bibliográficas}

BERGANZA, María Rosa y DEL HOYO, Mercedes (2006): "La mujer y el hombre en la publicidad televisiva: imágenes y estereotipos”. Zer, vol. 21. Bilbao, Servicio de Publicaciones Universidad del País Vasco, pp.161-175.

BUTLER-PAISLEY, Matilda y PAISLEY-BUTLER, William (1974), "Sexism in the media: frameworks for Research", paper presented at the Association for Education in Journalism, San Diego, CA.

CAMBRONERO, Belén, RUIZ-CANTERO, María Teresa y PAPÍ-GÁLVEZ, Natalia (2009): "Los estereotipos de género en la publicidad de fármacos". I Congreso Internacional Publicidad y Género. Congènere, Girona (actas).

FURNHAM, Adrian y BITAR, Nadine (1993): "The stereotyped portrayal of men and women in British television advertisements". Sex Roles, vol. 29. New York, Springer, pp. 297-310.

FURNHAM, Adrian y MAK, Twiggy (1999): "Sex-role stereotyping in television commercials: a review and comparison of fourteen studies done on five continents over 25 years". Sex Roles, vol. 41 (5/6), New York, Springer, pp.413-437.

GARCÍA-GONZÁLEZ, Aurora y PIÑEIRO-OTERO, Teresa (2011): "Las mujeres, en el ámbito de la producción publicitaria. Estudio del sector publicitario gallego desde una perspectiva de género", en Revista Latina de Comunicación Social: http://www.revistalatinacs.org/11/art/943_Galicia/22_Aurora.html [fecha de consulta: 31 de julio de 2012]

GILLY, Mary C. (1988): "Sex roles in advertising: a comparison of television advertisements in Australia, Mexico, and the United States". Journal of Marketing, vol. 52. Chicago, The American Marketing Association, pp. 75-85.

KIM, Kwangok y LOWRY, Dennis T. (2005): "Television commercials as a lagging social indicator: gender role stereotypes in Korean television advertising". Sex Roles, vol. 53, New York, Springer, pp. 901-910

MANSTEAD, A.S.R. y McCULLOCH, Caroline (1981): "Sex role stereotyping in British television advertisements". British Journal of Social Psychology, vol. 20. The British Psychological Society-Wiley, pp. 171-180. 
MARTÍN-LLAGUNO, Marta, BELÉNDEZ, Marina y HERNÁNDEZ, Alejandra (2007): La mujer en las agencias de publicidad. Madrid, AEAP.

MARTÍN-LLAGUNO, Marta y NAVARRO, Marián (2012): "Publicidad y leyes de violencia de género. Estudio empírico en España y Argentina". Questiones Publicitarias, vol. 1 (17). Universidad de Sevilla, Grupo de Investigación MAECEI, pp. 139-155.

McARTHUR, Leslie Z. y RESKO, Beth G. (1975): “The portrayal of men and women in American TV commercials". Journal of Social Psychology, vol. 97. London, Routledge, pp. 209-220.

MOON, Young Sook y CHAN, Kara (2002): "Gender portrayal in Hong Kong and Korean children's TV commercials. A cross-cultural comparison". Asian Journal of Communication, vol.12 (2). London, Asian Media Information and Communication Centre-Routdledge, pp. 100-119.

NETO, Félix y PINTO, Isabel (1998): “Gender stereotypes in Portuguese television advertisements”. Sex Roles, vol. 39. New York, Springer, pp. 153-164.

PINGREE, Suzanne, HAWKINS, Robert Parker, BUTLER, Matilda y PAISLEY, William (1976): "A scale for sexism". Journal of Communication, vol. 24. International Communication Association-Wiley, pp. 193-200.

PUEYO, Natalia (2010): "Sex structure of occupations in advertising industry: Where are the female ad practitioners?", en Observatorio (OBS*): http://obs.obercom.pt /index.php/obs/article/viewArticle/416. [fecha de consulta: 31 de julio de 2012]

ROYO-VELA, Mariano, ALDÁS-MANZANO, Joaquín, KÜSTER-BOLUDA, Inés y VILA-LOPEZ, Natalia (2007): "Gender role portrayals and sexism in Spanish magazines". Equal Opportunities International. Bingley, Emerald, vol. 26 (7), pp. 633-652.

ROYO-VELA, Mariano, ALDÁS-MANZANO, Joaquín, KÜSTER, Inés y VILA, Natalia (2008): "Adaptation of marketing activities to cultural and social context: gender role portrayals and sexism in Spanish commercials". Sex Roles, vol. 58. New York, Springer, pp. 379-390.

RUIZ-CANTERO, María Teresa, MARTÍN-LLAGUNO, Marta, LA PARRA, Daniel, VIVES, Carmen y ALBALADEJO, Manuel (2004): "El enfoque de género en las noticias de salud". Gaceta Sanitaria, 18 (supl.2). Elsevier, pp. 65-74.

URAY, Nimet y BURNAZ, Sebnem (2003): "An analysis of the portrayal of gender roles in Turkish television advertisements". Sex Roles, vol. 48. New York, Springer, pp. 77-87.

UTTERSTRÖM, Thomas (1977): "Sex discrimination in advertising: A summary of the situation in Sweden". Journal of Consumer Policy, vol.1. New York, Springer, pp. 359-372. 
Alejandra HERNÁNDEZ RUIZ

alejandra.hernandez@ua.es

Universidad de Alicante

Departamento de Comunicación y Psicología Social

Campus de Sant Vicent del Raspeig

Ap. 99 E-03080 Alicante

Profesora ayudante doctora de Deontología de la Publicidad y las RR.PP. y Gestión de la Información en Comunicación.

\section{Marta MARTÍN LLAGUNO}

marta.martin@ua.es

Universidad de Alicante

Departamento de Comunicación y Psicología Social

Campus de Sant Vicent del Raspeig

Ap. 99 E-03080 Alicante

Catedrática de Universidad de Fundamentos de la Comunicación II y Deontología de la Publicidad y las RR.PP.

\section{Marina BELÉNDEZ VÁZQUEZ}

marina.belendez@ua.es

Departamento de Comunicación y Psicología Social

Campus de Sant Vicent del Raspeig

Ap. 99 E-03080 Alicante

Profesora Titular de Universidad del área de Psicología Social. 